Our Nature
Journal homepage: http://nepjol.info/index.php/ON

\title{
Water quality and red bloom algae of fish ponds in three different regions of Nepal
}

\author{
Ram Bhajan Mandal ${ }^{{ }^{*}}$, Sunila Rai ${ }^{1}$, Madhav Kumar Shrestha ${ }^{1}$, Dilip Kumar Jha ${ }^{1}$, \\ Narayan Prasad Pandit ${ }^{1}$ and Shiva Kumar Rai ${ }^{2}$ \\ ${ }^{1}$ Agriculture and Forestry University, Rampur, Chitwan, Nepal \\ ${ }^{2}$ Department of Botany, Post Graduate Campus, Biratnagar, Nepal \\ *E-mail: rbmandal2008@gmail.com
}

\begin{abstract}
Present study determines the causes and seasonal variation of red bloom in fishponds of Eastern, Western and Central regions of Nepal. Monthly monitoring of water quality and phytoplankton was carried out for one year. Water parameters such as $\mathrm{NH}_{3}-\mathrm{N}$, total phosphorus, total Kjeldahl nitrogen (TKN), total dissolved solids (TDS) and conductivity were significantly higher $(\mathrm{p}<0.05)$ in red bloom fishponds than non-red bloom fishponds. The total density of euglenophytes in red-bloom fishponds was significantly higher $(\mathrm{P}<0.05)\left(1970 \pm 260\right.$ cells $\left.\mathrm{L}^{-1}\right)$ than non-red bloom fishponds $\left(410 \pm 30\right.$ cells $\left.\mathrm{L}^{-1}\right)$. Euglenophyte density varied seasonally and significantly lower in spring season $\left(1250 \pm 220\right.$ cells $\left.{ }^{-1}\right)$ than autumn $\left(1950 \pm 390\right.$ cells L $\left.{ }^{-1}\right)$, winter $\left(2180 \pm 370\right.$ cells L $\left.{ }^{-1}\right)$, and summer $\left(2490 \pm 480\right.$ cells L $\left.{ }^{-1}\right)$ in red bloom fishponds. High nutrients might favor the growth of euglenophytes (Euglena sanguinea) causing red bloom fish ponds of Nepal.
\end{abstract}

Key words: Conductivity, Euglenophytes, Fish production, Phytoplankton

DOI: http://dx.doi.org/10.3126/on.v14i1.16443

Manuscript details: Received: 02.11.2016 / Accepted: 27.11.2016

Citation: Mandal, R.B., S. Rai, M.K. Shrestha, D.K. Jha, N.P. Pandit and S.K. Rai 2016. Water quality and red bloom algae of fish ponds in three different regions of Nepal. Our Nature 14(1):71-77. DOI: http://dx.doi.org/10.3126/on.v14i1. 16443

Copyright: @ Mandal et al. 2016. Creative Commons Attribution-NonCommercial 4.0 International License.

\section{Introduction}

Red-bloom in fishponds is a common occurrence in Nepal. Red bloom fishponds are frequently covered with brick-red colored thin film of fine dust. This thin film or scum at the surface, which gives unpleasant look, shades the lower waters, inhibit photosynthesis, deplete dissolved oxygen, brings behavioral changes in fish and sometimes results fish mortality too (Rehman, 1998; Zimba et al., 2004; Zimba et al., 
2010) which is due to euglenoid toxin functions as a neurotoxin (Costa and Garrido, 2004; Costa, 2014). The color of the red bloom is not constant, that changes from green to red, generally green at the dawn and at the dusk, when the sunrays are oblique and soft (Rehman, 1998). Red blooms occur due to abundant food and suitable environment for phytoplankton growth.

For getting higher fish production, fish farmers feed their ponds with high doses of fertilizers. As a result, the confined water of the ponds becomes eutrophic due to sedimentation of nutrients from feed and fertilizers. This condition induces toxic and noxious phytoplankton bloom including euglenophytes which causes red blooming in pond water (Ohio EPA, 2013). Euglenophytes bloom is the most common phenomenon in warmer, shallow and eutrophic water bodies in Nepal. Euglena assemblages are known to be widely distributed in higher eutrophic shallow ponds at elevated temperature (Wild et al., 1995). Most problems occur when population of potentially toxic species increase dramatically and causes oxygen depletions killing fish, shellfish and other aquatic organisms (Lopez et al., 2008; Zimba et al., 2004; 2010; Boyd and Tucker, 2014). In Nepal, farmers generally believe that red bloom has adverse effects on fish farming such as low fish production, oxygen depletion and fish mortality; however, no scientific research is done yet to prove it. Therefore, present research was to assess causes and seasonal occurrence of red bloom in fish ponds in Nepal.

\section{Materials and methods}

Phytoplankton and water samples were collected from ten fishponds ( 5 red bloom and 5 non-red bloom ponds) each from 3 differ- ent regions (Morang in Eastern, Chitwan in Central and Rupandehi district in western region) of Nepal at 6 am to $8.30 \mathrm{am}$ in four different seasons (spring, autumn, summer and winter) in a year.

Water quality parameters such as dissolved oxygen (DO), $\mathrm{pH}$, oxidation reduction potential (ORP), temperature, conductivity, total dissolved solid (TDS) were analyzed in situ using HI-98194 Multiparameter and soluble reactive phosphorus (SRP), nitrate, nitrite, ammonia nitrogen $\left(\mathrm{NH}_{3}-\mathrm{N}\right)$ using HI-83203-02 Multiparameter bench photometer. Total phosphorus and chlorophyll-a was analyzed by standard methods (APHA, 1985; 2012) and total Kjheldahl nitrogen (TKN) according to APHA (2005).

For phytoplankton sampling, five liters of pond water (up to $50 \mathrm{~cm}$ depth) was collected using graduated bucket and filtered with plankton net (mesh size $5 \mu \mathrm{m}$ ) and then preserved in 5\% formaldehyde solution. Phytoplanktons were identified following Prescott (1951) and Rai and Rai (2007) and classified according to Guiry and Guiry (2016). Phytoplankton were counted using Sedgwick-Rafter (S-R) cells and quantified following APHA (1976) as.

Number of species $=\mathrm{C} \times 1000 \mathrm{~mm}^{3} / \mathrm{L} \times \mathrm{D}$ $\mathrm{x} \mathrm{W} \times \mathrm{S}$

Where, $C=$ Number of organisms counted, $\mathrm{L}=$ Length of each stripe $(\mathrm{mm}), \mathrm{D}=$ Depth of each stripe $(\mathrm{mm}), \mathrm{W}=$ Width of each stripe $(\mathrm{mm})$, and $S=$ Number of stripes.

Data was analyzed by one way ANOVA and significant level was considered at the level of $5 \%(\mathrm{P}<0.05)$. Seasonal variation and intra-regional of red bloom fishpond and non-red fishponds were analyzed by independent simple t-test at the level of $5 \%(\mathrm{P}<0.05)$. 


\section{Results}

Results of region wise water quality parameters and phytoplankton density is shown in Table 1 and season wise water quality parameters and phytoplankton density is shown in Table 2. Results showed that water quality parameter such as $\mathrm{pH}$, temperature, Nitrite, ORP and chlorophyll-a were not significantly different $(\mathrm{P}>0.05)$ between red bloom and non-red bloom fishponds.

\section{Region wise water quality parameters and phytoplankton density}

Dissolved oxygen was of significantly lower $(2.3 \pm 0.5 \mathrm{mg} / \mathrm{L})$ in red bloom fishponds of central region than non-red bloom fishponds of $(3.1 \pm 0.3 \mathrm{mg} / \mathrm{L})$ the same region (Tab.1). Total phosphorus in red bloom fishponds was significantly higher $(1.16 \pm 0.28 \mathrm{mg} / \mathrm{L})$ in eastern region than non-red bloom fishponds $(0.55 \pm 0.16 \mathrm{mg} / \mathrm{L})$ of same region (Tab.1). Total ammonia nitrogen $\left(\mathrm{NH}_{3}-\mathrm{N} \mathrm{mg} / \mathrm{L}\right)$ in red bloom fishponds of eastern region was also significantly higher $(1.48 \pm 0.30 \mathrm{mg} / \mathrm{L})$ than nonred bloom fishponds $(0.63 \pm 0.09 \mathrm{mg} / \mathrm{L})$ of same region (Tab. 1). Total Kjheldahl nitrogen (TKN) of red bloom fishponds of eastern $(1.55 \pm 0.12 \mathrm{mg} / \mathrm{L})$, central $(1.4 \pm 0.19$ $\mathrm{mg} / \mathrm{L})$ and western $(1.48 \pm 0.07 \mathrm{mg} / \mathrm{L}) \mathrm{re}-$ gions were significantly higher $(\mathrm{P}<0.05)$ than non-red bloom fishponds of all three different regions (Tab. 1). Similarly, TDS and conductivity of red bloom fishponds of all three regions were significantly higher $(\mathrm{P}<0.05)$ than non-red bloom fishponds. Highest value of TDS and conductivity were observed in western regions of Nepal (Tab. 1). Euglenophytes was significantly higher $(\mathrm{P}<0.05)$ in red bloom fishponds of western region $\left(2370 \pm 420\right.$ cells $\left.\mathrm{L}^{-1}\right)$ than non-red bloom fishponds $\left(490 \pm 70\right.$ cells $\left.\mathrm{L}^{-1}\right)$ of the same region (Tab. 1). Bascillario- phytes and cyanophytes were not significantly different $(\mathrm{P}>0.05)$ among red bloom or non-red bloom fish ponds of all three regions (Tab. 1).

\section{Season wise water quality parameters and phytoplankton density}

Seasonal combined mean DO (combined mean value of all red bloom fishponds or non-red bloom fishponds) was significantly higher in non-red bloom fishpond (3.5 \pm 0.4 $\mathrm{mg} / \mathrm{L})$ than red bloom fishponds $(2.5 \pm 0.1$ $\mathrm{mg} / \mathrm{L}$ ) (Tab. 2). Combined mean of total phosphorus also found significantly higher in red bloom fishponds $(0.86 \pm 0.12 \mathrm{mg} / \mathrm{L})$ than non-red bloom fish ponds $(0.49 \pm 0.05$ $\mathrm{mg} / \mathrm{L}$ ) (Tab. 2). Similarly combined mean of $\mathrm{NH}_{3}-\mathrm{N} \mathrm{mg} / \mathrm{L}$ in red bloom fishponds was significantly higher $(1.05 \pm 0.14 \mathrm{mg} / \mathrm{L})$ than non-red bloom fishponds $(0.64 \pm 0.08$ $\mathrm{mg} / \mathrm{L}$ ). Combined mean value of total dissolve solids (TDS) of red bloom fish ponds was significantly higher $(148.01 \pm 14.5$ $\mathrm{mg} / \mathrm{L}$ ) than non-red bloom fishponds (105.01 $\pm 13.10 \mathrm{mg} / \mathrm{L})$ (Tab. 2). Similarly, combined conductivity was also significantly higher in red bloom fishponds $(292.3 \pm 31.1 \mu \mathrm{S} / \mathrm{cm})$ than non-red bloom fishponds (211.98 $\pm 27.2 \mu \mathrm{S} / \mathrm{cm})$ (Tab. 2). Combined mean nitrate value in non-red bloom fishponds was significantly higher $(0.59 \pm 0.09 \mathrm{mg} / \mathrm{L})$ than red bloom fishponds $(0.36 \pm 0.03 \mathrm{mg} / \mathrm{L})$.

Euglenophytes was significantly higher $(\mathrm{P}<0.05)$ in summer season $\left(2490 \pm 290\right.$ cells $\left.\mathrm{L}^{-1}\right)$, winter $(2180 \pm 370$ cells $\left.\mathrm{L}^{-1}\right)$ and autumn $\left(1950 \pm 390\right.$ cells $\left.\mathrm{L}^{-1}\right)$, than spring $\left(1250 \pm 220\right.$ cells $\left.\mathrm{L}^{-1}\right)$ season (Tab. 2) in red bloom fishponds. Chlorophytes was found significantly higher in summer season $\left(1620 \pm 250\right.$ cells $\left.\mathrm{L}^{-1}\right)$ than autumn season $\left(650 \pm 60\right.$ cells $\left.\mathrm{L}^{-1}\right)$ in red 
Mandal et al. / Our Nature (2016), 14 (1): 71-77

Table 1. Region-wise variation of water quality parameters and phytoplankton (Mean + S.E.) of red bloom and non-red bloom fishponds.

\begin{tabular}{|c|c|c|c|c|c|c|}
\hline \multirow[t]{2}{*}{ Parameter } & \multicolumn{2}{|c|}{ Eastern Nepal } & \multicolumn{2}{|c|}{ Central Nepal } & \multicolumn{2}{|l|}{ Western Nepal } \\
\hline & Red bloom & $\begin{array}{l}\text { Non-Red } \\
\text { bloom }\end{array}$ & Red bloom & $\begin{array}{l}\text { Non-Red } \\
\text { bloom }\end{array}$ & Red bloom & $\begin{array}{l}\text { Non-Red } \\
\text { bloom }\end{array}$ \\
\hline Temperature $\left({ }^{\circ} \mathrm{C}\right)$ & $28.70 \pm 0.1^{\mathrm{aA}}$ & $28.32 \pm 0.1^{\mathrm{aA}}$ & $26.0 \pm 0.3^{\mathrm{bA}}$ & $25.77 \pm 0.3^{\mathrm{bA}}$ & $25.80 \pm 0.2^{\mathrm{bA}}$ & $25.96 \pm 0.2^{\mathrm{bA}}$ \\
\hline $\mathrm{pH}$ & $7.7(7.4-8.0)$ & $7.4(7.4-7.8)$ & $7.8(7.7-.8 .0)$ & $7.6(7.3-8.2)$ & $7.9(7.7-8.2)$ & $7.8(7.6-8.4)$ \\
\hline $\mathrm{DO}(\mathrm{mg} / \mathrm{L})$ & $3.0 \pm 0.2^{\mathrm{bB}}$ & $3.8 \pm 0.2^{\mathrm{aA}}$ & $2.3 \pm 0.5^{\mathrm{cB}}$ & $3.1 \pm 0.3^{\mathrm{bA}}$ & $2.4 \pm 0.4^{\mathrm{cB}}$ & $3.7 \pm 0.5^{\mathrm{aA}}$ \\
\hline ORP (mV) & $\begin{array}{l}110.55 \pm 12.1^{\mathrm{a}} \\
\mathrm{A}\end{array}$ & $134.85 \pm 5.43^{\mathrm{aA}}$ & $\begin{array}{l}124.35 \pm 2.83^{\mathrm{a}} \\
\mathrm{A}\end{array}$ & $131.37 \pm 3.0^{\mathrm{aA}}$ & $125.85 \pm 5.41^{\mathrm{aA}}$ & $131.67 \pm 6.05^{\mathrm{aA}}$ \\
\hline $\mathrm{NH}_{3}-\mathrm{N}(\mathrm{mg} / \mathrm{L})$ & $1.48 \pm 0.30^{\mathrm{aA}}$ & $0.63 \pm 0.09^{\mathrm{bB}}$ & $0.78 \pm 0.19^{\mathrm{bA}}$ & $0.53 \pm 0.16^{\mathrm{bA}}$ & $0.91 \pm 0.18^{\mathrm{bA}}$ & $0.77 \pm 0.18^{\mathrm{bA}}$ \\
\hline $\mathrm{SRP}(\mathrm{mg} / \mathrm{L})$ & $0.25 \pm 0.06^{\mathrm{aA}}$ & $0.29 \pm 0.06^{\mathrm{aA}}$ & $0.24 \pm 0.06^{\mathrm{aA}}$ & $0.15 \pm 0.02^{\mathrm{aB}}$ & $0.24 \pm 0.07^{\mathrm{aA}}$ & $0.21 \pm 0.07^{\mathrm{aA}}$ \\
\hline Nitrate $(\mathrm{mg} / \mathrm{L})$ & $0.41 \pm 0.1^{\mathrm{aA}}$ & $0.52 \pm 0.16^{\mathrm{aA}}$ & $0.28 \pm 0.09^{\mathrm{aB}}$ & $0.57 \pm 0.28{ }^{\mathrm{aA}}$ & $0.4 \pm 0.17^{\mathrm{aA}}$ & $0.69 \pm 0.29^{\mathrm{aA}}$ \\
\hline Nitrite $(\mathrm{mg} / \mathrm{L})$ & $0.01 \pm 0.00^{\mathrm{bA}}$ & $0.01 \pm 0.00^{\mathrm{bA}}$ & $0.09 \pm 0.03^{\mathrm{bA}}$ & $0.05 \pm 0.02^{\mathrm{bA}}$ & $0.28 \pm 0.08^{\mathrm{aA}}$ & $0.22 \pm 0.02^{\mathrm{aA}}$ \\
\hline Ch-a (mg/L) & $22.3 \pm 3.07^{\mathrm{aA}}$ & $32.05 \pm 5.30^{\mathrm{aA}}$ & $\begin{array}{l}30.76 \pm 3.84 \\
\text { aA }\end{array}$ & $27.51 \pm 4.20^{\mathrm{aA}}$ & $33.79 \pm 1.57^{\mathrm{aA}}$ & $28.57 \pm 3.64^{\mathrm{aA}}$ \\
\hline TDS (mg/L) & $\begin{array}{l}100.5 \pm 6.65 \\
\mathrm{cA}\end{array}$ & $\begin{array}{l}60.81 \pm 4.42 \\
\text { eB }\end{array}$ & $\begin{array}{l}134.1 \pm 7.32 \\
\mathrm{cA}\end{array}$ & $84.57 \pm 11.24^{\mathrm{cdB}}$ & $\begin{array}{l}209.43 \pm 23.60^{\mathrm{a}} \\
\text { A }\end{array}$ & $\begin{array}{l}169.64 \pm 4.61 \\
\text { bB }\end{array}$ \\
\hline $\begin{array}{l}\text { Conductivity } \\
(\mu \mathrm{S} / \mathrm{cm})\end{array}$ & $\begin{array}{l}187.7 \pm 7.48 \\
\text { dA }\end{array}$ & $123.60 \pm 8.43^{\mathrm{dB}}$ & $\begin{array}{l}267.34 \pm 14.9^{\mathrm{c}} \\
\text { A }\end{array}$ & $\begin{array}{l}165.35 \pm 23.5 \\
d B\end{array}$ & $\begin{array}{l}421.87 \pm 53.06^{\mathrm{a}} \\
\text { A }\end{array}$ & $\begin{array}{l}347.0 \pm 9.65 \\
\text { bB }\end{array}$ \\
\hline TKN (mg/L) & $1.55 \pm 0.12^{\mathrm{aA}}$ & $1.1 \pm 0.07^{\mathrm{bcB}}$ & $1.4 \pm 0.19^{\mathrm{abA}}$ & $1.00 \pm 0.07^{\mathrm{cB}}$ & $1.48 \pm 0.1^{\mathrm{aA}}$ & $1.07 \pm 0.06^{\mathrm{bcB}}$ \\
\hline TP (mg/L) & $1.16 \pm 0.28^{\mathrm{aA}}$ & $0.55 \pm 0.16^{\mathrm{bA}}$ & $0.59 \pm 0.12^{\mathrm{bA}}$ & $0.36 \pm 0.06^{\mathrm{bA}}$ & $0.83 \pm 0.14^{\mathrm{abA}}$ & $0.56 \pm 0.06^{\mathrm{bA}}$ \\
\hline Phytoplankton & & & & & & \\
\hline $\begin{array}{l}\text { Euglenophytes } \\
\left(\mathrm{X} 10^{3} \text { cells } / \mathrm{L}\right)\end{array}$ & $1.36 \pm 0.08^{\mathrm{abA}}$ & $\begin{array}{l}0.42 \pm 0.01 \\
\operatorname{cdB}\end{array}$ & $\begin{array}{l}2.19 \pm 0.62 \\
\text { aA }\end{array}$ & $\begin{array}{l}0.34 \pm 0.06 \\
\mathrm{~dB}\end{array}$ & $\begin{array}{l}2.37 \pm 0.42 \\
\text { aA }\end{array}$ & $\begin{array}{l}0.49 \pm 0.07 \\
\mathrm{cdB}\end{array}$ \\
\hline $\begin{array}{l}\text { Chlorophytes } \\
\left(\mathrm{X} 10^{3} \text { cells } / \mathrm{L}\right)\end{array}$ & $1.01 \pm 0.14^{\mathrm{bcA}}$ & $\begin{array}{l}1.29 \pm 0.19 \\
\text { abcA }\end{array}$ & $\begin{array}{l}1.5 \pm 0.07 \\
\mathrm{abA}\end{array}$ & $\begin{array}{l}1.55 \pm 0.29 \\
\text { aA }\end{array}$ & $\begin{array}{l}0.83 \pm 0.09 \\
\mathrm{cA}\end{array}$ & $\begin{array}{l}1.3 \pm 0.08 \\
\text { abcA }\end{array}$ \\
\hline $\begin{array}{l}\text { Bascillariophytes } \\
\left(\mathrm{X} 10^{3} \text { cells/L) }\right.\end{array}$ & $\begin{array}{l}0.58 \pm 0.19 \\
\text { aA }\end{array}$ & $\begin{array}{l}0.39 \pm 0.07 \\
\text { aA }\end{array}$ & $\begin{array}{l}0.48 \pm 0.07 \\
\text { aA }\end{array}$ & $\begin{array}{l}0.53 \pm 0.14 \\
\text { aA }\end{array}$ & $\begin{array}{l}0.50 \pm 0.13 \\
\text { aA }\end{array}$ & $\begin{array}{l}0.52 \pm 0.14 \\
\text { aA }\end{array}$ \\
\hline $\begin{array}{l}\text { Cyanophytes } \\
\left(\mathrm{X} 10^{3} \text { cells/L) }\right.\end{array}$ & $\begin{array}{l}0.39 \pm 0.08 \\
\text { bA }\end{array}$ & $\begin{array}{l}0.63 \pm 0.10 \\
\text { aA }\end{array}$ & $\begin{array}{l}0.30 \pm 0.02 \\
\mathrm{bA}\end{array}$ & $\begin{array}{l}0.39 \pm 0.08 \\
\text { bA }\end{array}$ & $\begin{array}{l}0.22 \pm 0.01 \\
\mathrm{bA}\end{array}$ & $\begin{array}{l}0.39 \pm 0.08 \\
\mathrm{bA}\end{array}$ \\
\hline
\end{tabular}

[Small letter shows regional differences while capital letter (t-test analysis) shows intra-regional differences between red bloom and non-red bloom fishponds] 
Mandal et al. / Our Nature (2016), 14 (1): 71-77

Table 2. Season-wise variation of water quality parameters and phytoplankton of red bloom and non-red bloom fishponds in all three regions of country.

\begin{tabular}{|c|c|c|c|c|c|c|c|c|c|c|}
\hline \multirow[b]{2}{*}{ Parameter } & \multicolumn{2}{|c|}{ Spring(M-M) } & \multicolumn{2}{|c|}{ Summer (J -A) } & \multicolumn{2}{|c|}{ Autumn (S -N) } & \multicolumn{2}{|c|}{ Winter (D - F) } & \multicolumn{2}{|c|}{ Combined } \\
\hline & $\begin{array}{l}\text { Red } \\
\text { pond }\end{array}$ & $\begin{array}{l}\text { Non- } \\
\text { Red }\end{array}$ & $\begin{array}{l}\text { Red } \\
\text { pond }\end{array}$ & $\begin{array}{l}\text { Non- } \\
\text { Red }\end{array}$ & $\begin{array}{l}\text { Red } \\
\text { pond }\end{array}$ & $\begin{array}{l}\text { Non- } \\
\text { Red }\end{array}$ & $\begin{array}{l}\text { Red } \\
\text { pond }\end{array}$ & $\begin{array}{l}\text { Non- } \\
\text { Red }\end{array}$ & $\begin{array}{l}\text { Red } \\
\text { pond }\end{array}$ & $\begin{array}{l}\text { Non- } \\
\text { Red }\end{array}$ \\
\hline & $\begin{array}{l}30.0 \pm 0 . \\
9^{\text {a }}\end{array}$ & & $\begin{array}{l}31.8 \pm 0 . \\
1^{\mathrm{a}}\end{array}$ & $\begin{array}{l}31.3 \pm 0 . \\
01 \quad \mathrm{~b}\end{array}$ & $6^{\mathrm{a}}$ & $\begin{array}{l}26.1 \pm 0 . \\
6^{\mathrm{a}}\end{array}$ & $2^{a}$ & $3^{a}$ & $\begin{array}{l}20.8 \\
3^{a}\end{array}$ & \\
\hline $\mathrm{H}$ & & $7.8 \pm 0.1^{\mathrm{a}}$ & & & & & & $\begin{array}{l}7.9 \pm 0.0 \\
7^{\mathrm{a}}\end{array}$ & & \\
\hline $\mathrm{m}$ & & $\begin{array}{l}3.9 \pm 0.44 \\
\mathrm{a}\end{array}$ & & $2.4 \pm 0.2^{\mathrm{a}}$ & $2.3 \pm 0.2^{b}$ & $\begin{array}{l}3.1 \pm 0.3 \\
2^{\mathrm{a}}\end{array}$ & & & & $3.5 \pm 0.4^{\mathrm{a}}$ \\
\hline PD & $\begin{array}{l}106.52 \pm \\
6.6^{\mathrm{a}}\end{array}$ & $\begin{array}{l}119.17 \pm \\
6.81^{\mathrm{a}}\end{array}$ & $\begin{array}{l}113.59 \pm \\
5.7^{\mathrm{a}}\end{array}$ & $\begin{array}{l}124.1 \pm 3 \\
.4^{\mathrm{a}}\end{array}$ & & $\begin{array}{l}147.59 \pm \\
3.39^{\mathrm{a}}\end{array}$ & $\begin{array}{l}133.62 \pm \\
8.4^{\mathrm{a}}\end{array}$ & $\begin{array}{l}139.69 \pm \\
5.9^{a}\end{array}$ & & $\begin{array}{l}132.63 \pm \\
2.89^{a}\end{array}$ \\
\hline $\mathrm{SH}$ & $\begin{array}{l}0.91 \pm 0 . \\
19^{\mathrm{a}}\end{array}$ & $\begin{array}{l}0.64 \pm 0.1 \\
6^{\mathrm{a}}\end{array}$ & $\begin{array}{l}1.21 \pm 0 . \\
19^{\mathrm{a}}\end{array}$ & $\begin{array}{l}0.42 \pm 0 \\
06^{\mathrm{b}}\end{array}$ & $\begin{array}{l}1.05 \pm 0 . \\
20^{\mathrm{a}}\end{array}$ & $\begin{array}{l}0.75 \pm 0 . \\
13^{\mathrm{a}}\end{array}$ & $\begin{array}{l}1.01 \pm 0 . \\
22^{\mathrm{a}}\end{array}$ & $\begin{array}{l}0.76 \pm 0 \\
17^{\mathrm{a}}\end{array}$ & $\begin{array}{l}1.05 \pm 0 . \\
14^{\mathrm{a}}\end{array}$ & $\begin{array}{l}0.64 \pm 0.0 \\
8^{b}\end{array}$ \\
\hline SRP ( & $\begin{array}{l}0.13 \pm 0 . \\
03^{\mathrm{a}}\end{array}$ & $\begin{array}{l}0.19 \pm 0.0 \\
6^{\mathrm{a}}\end{array}$ & $\begin{array}{l}0.29 \pm 0 \\
07^{\mathrm{a}}\end{array}$ & $\begin{array}{l}0.13 \pm 0 \\
03^{\mathrm{a}}\end{array}$ & $\begin{array}{l}0.31 \pm 0 . \\
08^{\mathrm{a}}\end{array}$ & $\begin{array}{l}0.34 \pm 0 . \\
08^{\mathrm{a}}\end{array}$ & $\begin{array}{l}0.24 \pm 0 . \\
05^{\text {a }}\end{array}$ & $\begin{array}{l}0.19 \pm 0 . \\
066^{\mathrm{a}}\end{array}$ & $\begin{array}{l}0.24 \pm 0 \\
033^{\mathrm{a}}\end{array}$ & \\
\hline Jitro & $\begin{array}{l}4 \pm 0 . \\
\mathrm{a}\end{array}$ & $\begin{array}{l}0.91 \pm 0.2 \\
1^{\mathrm{a}}\end{array}$ & $\begin{array}{l}0.19 \pm 0 . \\
04^{\mathrm{a}}\end{array}$ & $\begin{array}{l}0.4 \pm 0.1 \\
7^{\mathrm{a}}\end{array}$ & $\begin{array}{l}0.1 \pm 0.0 \\
4^{b}\end{array}$ & $\begin{array}{l}0.49 \pm 0 . \\
16^{\mathrm{a}}\end{array}$ & $\begin{array}{l}0.32 \pm 0 . \\
1^{\mathrm{a}}\end{array}$ & $\begin{array}{l}0.57 \pm 0 . \\
15^{\text {a }}\end{array}$ & $\begin{array}{l}0.36 \pm 0 \\
03^{\mathrm{b}}\end{array}$ & $\begin{array}{l}0.59 \pm 0.0 \\
9^{\mathrm{a}}\end{array}$ \\
\hline & $\begin{array}{l}0.06 \pm 0 \\
02^{\mathrm{a}}\end{array}$ & $\begin{array}{l}0.03 \pm 0.0 \\
1^{\mathrm{a}}\end{array}$ & $\begin{array}{l}0.01 \pm 0 . \\
0^{\mathrm{a}}\end{array}$ & $\begin{array}{l}0.02 \pm 0 . \\
0^{\mathrm{a}}\end{array}$ & $\begin{array}{l}0.02 \pm 0 . \\
00^{\mathrm{a}}\end{array}$ & $\begin{array}{l}0.03 \pm 0 . \\
0^{\mathrm{a}}\end{array}$ & $\begin{array}{l}0.43 \pm 0 . \\
14^{\mathrm{a}}\end{array}$ & $\begin{array}{l}0.29 \\
1^{\mathrm{a}}\end{array}$ & $\begin{array}{l}0.13 \pm 0 \\
04^{\mathrm{a}}\end{array}$ & $\begin{array}{l}0.09 \pm \\
2^{\mathrm{a}}\end{array}$ \\
\hline & $\begin{array}{l}21.9 \pm 4 . \\
06^{\mathrm{a}}\end{array}$ & $\begin{array}{l}29.44 \pm 4 . \\
44^{\mathrm{a}}\end{array}$ & $\begin{array}{l}30.7 \\
.35\end{array}$ & $\begin{array}{l}37.98 \pm 5 \\
.6^{\mathrm{a}}\end{array}$ & $\begin{array}{l}23.07 \pm 3 \\
.3^{\mathrm{a}}\end{array}$ & $\begin{array}{l}21.68 \pm 2 \\
.61^{\mathrm{a}}\end{array}$ & $\begin{array}{l}40.07 \pm 6 \\
.5^{\mathrm{a}}\end{array}$ & $\begin{array}{l}28.42 \\
.3^{\mathrm{a}}\end{array}$ & & $\begin{array}{l}29.38 \\
42^{a}\end{array}$ \\
\hline & $\begin{array}{l}160.07 \pm \\
18.9^{\mathrm{a}}\end{array}$ & $\begin{array}{l}11 \\
16\end{array}$ & $\begin{array}{l}106 \\
15 .\end{array}$ & & & $\begin{array}{l}99.9 \pm 14 \\
.9^{\mathrm{a}}\end{array}$ & & & & \\
\hline & $\begin{array}{l}298.73 \pm \\
43.3^{\mathrm{a}}\end{array}$ & & & $\begin{array}{l}169.96 \pm \\
24.3^{\mathrm{a}}\end{array}$ & $\begin{array}{l}28 \\
33\end{array}$ & $\begin{array}{l}203 . \\
29.9\end{array}$ & & & & $\begin{array}{l}211.98 \pm \\
27.2^{\mathrm{b}}\end{array}$ \\
\hline & $\begin{array}{l}1.45 \pm 0 . \\
15^{\mathrm{a}}\end{array}$ & $\begin{array}{l}1.12 \pm 0.1 \\
1^{\mathrm{a}}\end{array}$ & $\begin{array}{l}1.59 \pm 0 . \\
1^{\mathrm{a}}\end{array}$ & $\begin{array}{l}1.39 \pm 0 . \\
06^{\mathrm{a}}\end{array}$ & $\begin{array}{l}1.24 \pm 0 . \\
13^{\mathrm{a}}\end{array}$ & $\begin{array}{l}0.88 \pm 0 . \\
11^{\mathrm{b}}\end{array}$ & $\begin{array}{l}1.62 \pm 0 . \\
25^{\mathrm{a}}\end{array}$ & $2 \pm 0$ & $\begin{array}{l}1.48 \pm 0 \\
07^{\mathrm{a}}\end{array}$ & $\begin{array}{l}1.05 \\
3^{\mathrm{b}}\end{array}$ \\
\hline & $\begin{array}{l}1.01 \pm 0 . \\
16^{\mathrm{a}}\end{array}$ & $\begin{array}{l}0.62 \pm 0.0 \\
9^{\mathrm{a}}\end{array}$ & $\begin{array}{l}0.74 \pm 0 . \\
2^{\mathrm{a}}\end{array}$ & $\begin{array}{l}0.38 \pm 0 \\
06^{\mathrm{a}}\end{array}$ & $\begin{array}{l}0.76 \pm 0 \\
17^{\mathrm{a}}\end{array}$ & $\begin{array}{l}0.40 \pm 0 . \\
08^{\text {a }}\end{array}$ & $\begin{array}{l}0.94 \pm 0 . \\
17^{\text {a }}\end{array}$ & $\begin{array}{l}0.55 \pm 0 . \\
08^{\mathrm{b}}\end{array}$ & $\begin{array}{l}0.86 \pm 0 \\
12^{\mathrm{a}}\end{array}$ & $\begin{array}{l}0.49 \pm \\
5^{\mathrm{b}}\end{array}$ \\
\hline & & & & & & & & & & \\
\hline & $\begin{array}{l}1.25 \pm 0 . \\
22^{\mathrm{aB}}\end{array}$ & $\begin{array}{l}0.58 \pm 0.0 \\
5^{\mathrm{bX}}\end{array}$ & $\begin{array}{l}2.49 \pm 0 \\
48^{\mathrm{aA}}\end{array}$ & $\begin{array}{l}0.29 \pm 0 . \\
04^{\mathrm{bY}}\end{array}$ & $\begin{array}{l}1.95 \pm 0 . \\
39^{\mathrm{aA}}\end{array}$ & $\begin{array}{l}0.24 \pm 0 . \\
04^{\text {bY }}\end{array}$ & $\begin{array}{l}2.18 \pm 0 . \\
37^{\text {aA }}\end{array}$ & $\begin{array}{l}0.56 \pm 0 \\
07^{\mathrm{bX}}\end{array}$ & $\begin{array}{l}1.97 \pm 0 . \\
26^{\mathrm{a}}\end{array}$ & $\begin{array}{l}0.41 \pm 0.0 \\
3^{\mathrm{b}}\end{array}$ \\
\hline $\begin{array}{l}\text { Chlorophytes } \\
\left(\mathrm{X} 10^{3} \text { cells/L) }\right.\end{array}$ & $\begin{array}{l}1.19 \pm 0 . \\
17^{\mathrm{aAB}}\end{array}$ & $\begin{array}{l}1.13 \pm 0.2 \\
\mathrm{bY}\end{array}$ & $\begin{array}{l}1.62 \pm 0 . \\
25^{\mathrm{aA}}\end{array}$ & $\begin{array}{l}2.04 \pm 0 . \\
26^{\mathrm{aX}}\end{array}$ & $\begin{array}{l}0.65 \pm 0 . \\
06^{\mathrm{bC}}\end{array}$ & $\begin{array}{l}1.09 \pm 0 . \\
18^{\mathrm{aY}}\end{array}$ & $\begin{array}{l}0.97 \pm 0 . \\
14^{\mathrm{aBC}}\end{array}$ & $\begin{array}{l}1.25 \pm 0 . \\
17^{\text {aT }}\end{array}$ & $\begin{array}{l}1.11 \pm 0 . \\
09^{\mathrm{a}}\end{array}$ & $\begin{array}{l}1.37 \pm 0.1 \\
1^{\mathrm{a}}\end{array}$ \\
\hline $\begin{array}{l}\text { Bascillariophytes } \\
\left(\mathrm{X} 10^{3} \text { cells/L) }\right.\end{array}$ & $\begin{array}{l}0.49 \pm 0 . \\
05^{\mathrm{aAB}}\end{array}$ & $\begin{array}{l}0.34 \pm 0.0 \\
3^{\mathrm{bY}}\end{array}$ & $\begin{array}{l}0.72 \pm 0 . \\
15^{\text {aA }}\end{array}$ & $\begin{array}{l}0.92 \pm 0 . \\
17^{\mathrm{aX}}\end{array}$ & $\begin{array}{l}0.37 \pm 0 . \\
05^{\mathrm{aC}}\end{array}$ & $\begin{array}{l}0.32 \pm 0 . \\
05^{\mathrm{aY}}\end{array}$ & $\begin{array}{l}0.49 \pm 0 . \\
07^{\mathrm{aAB}}\end{array}$ & $\begin{array}{l}0.33 \pm 0 . \\
04^{\text {aY }}\end{array}$ & $\begin{array}{l}0.52 \pm 0 . \\
06^{\mathrm{a}}\end{array}$ & $\begin{array}{l}0.48 \pm 0.0 \\
5^{\mathrm{a}}\end{array}$ \\
\hline $\begin{array}{l}\text { Cyanophytes } \\
\left(\mathrm{X}\left(10^{3} \text { cells/L) }\right.\right.\end{array}$ & $\begin{array}{l}0.33 \pm 0 \\
03^{\mathrm{aAB}}\end{array}$ & $\begin{array}{l}0.27 \pm 0.0 \\
3^{\text {aY }}\end{array}$ & $\begin{array}{l}0.43 \pm 0 . \\
1^{\mathrm{bA}}\end{array}$ & $\begin{array}{l}0.98 \pm 0 . \\
18^{\mathrm{aX}}\end{array}$ & $\begin{array}{l}0.18 \pm 0 . \\
02^{\mathrm{bB}}\end{array}$ & $\begin{array}{l}0.30 \pm 0 . \\
05^{\mathrm{aY}}\end{array}$ & $\begin{array}{l}0.27 \pm 0 . \\
03^{\mathrm{aAB}}\end{array}$ & $\begin{array}{l}0.32 \pm 0 \\
04^{\mathrm{aY}}\end{array}$ & $\begin{array}{l}0.30 \pm 0 . \\
03^{\mathrm{b}}\end{array}$ & $\begin{array}{l}0.47 \pm 0.0 \\
5^{\mathrm{a}}\end{array}$ \\
\hline
\end{tabular}

[Small letter shows difference between red bloom and non-red bloom fishponds while capital letter shows seasonal differences between red bloom and non-red bloom fishponds] 
bloom fishponds (Tab. 2). Cyanophytes was found significantly higher in non-red bloom fishponds $\left(980 \pm 180\right.$ cells $\left.\mathrm{L}^{-1}\right)$ in summer season than non-red bloom fishponds $\left(270 \pm 30\right.$ cells $\left.\mathrm{L}^{-1}\right)$ of the spring season. Bascillariophytes was significantly higher non-red bloom fishponds $(920 \pm 170$ cells $\mathrm{L}^{-1}$ ) in summer season than autumn $\left(320 \pm 50\right.$ cells L $\left.{ }^{-1}\right)$ (Tab. 2) season of nonred bloom fishponds. In red bloom fishponds, red color was depended on the presence of Euglena sanguinea. At least many than thousands cells per liter of water was found and if number of cells increased, intensity of red blooming also increased.

\section{Discussion}

Significantly higher euglenophyte density in red bloom fish ponds confirmed red bloom is due to euglenophytes (Rahman et al., 2007). The development of red bloom algae i.e., euglenophytes depend on the combination of a set of factors such as sunlight, temperature and nutrient concentrations. In accordance with Nwankwo (1995), higher number of euglenophytes species were recorded when water nutrient values were high. In the red bloom fishponds of all three regions during summer and winter seasons, the high nutrient such as TP, TKN, $\mathrm{NH}_{3}-\mathrm{N}$ was found which is quite similar to Xavier et al. (1991) and Rahman et al. (2007). The value of dissolved oxygen during the heavy bloom in ponds of the Central and Western region were lower (2.2 and 2.4 mg L-1) than the ponds of Eastern region $3.0 \mathrm{mg} \mathrm{L}-1$ where the bloom did not occur high. High dissolved solids (TDS), high SRP, TKN and high conductivity were found to favor the abundance of euglenophytes in Western and Central regions of Nepal (Munawar, 1970; Rahman et al., 2007). Nutrients enrichment in fishponds is reflected through TDS and conductivity of water.

This finding agrees fairly well with finding of Mishra and Saksena (1993) and Hosmani (1988) who reported that the percentage of euglenophytes was greater compared to chlorophytes, bacillariophytes and cyanophytes in nutrient rich water bodies (Costa and Garrido, 2004; Lopez et al., 2008; Costa, 2014).

\section{Conclusions}

Red algal bloom have increased during the past 20 years in Asian country (Costa and Garrido, 2004; Lopez et al., 2008; Costa, 2014). In Nepal, red bloom in fish ponds has become common problem especially in the terai region. Preliminary study on red bloom in fish ponds of three regions of the country has been able to identify that euglenophyte algae is the major cause of red scum and its population varies seasonally depending on nutrients content in water. The findings will help to make farmers understand that red color in fish pond is a biological activity influenced by water quality such as nutrients in water. However, further studies are needed to assess effects of red bloom on fish growth and production and its control measures.

\section{Acknowledgements}

Authors are thankful to the farmers of Eastern, Central and Western regions of Nepal for their kind cooperation and help during the field study. We would also like to thank Department of Aquaculture and Fisheries, Agriculture and Fisheries University, and Department of Botany, Postgraduate Campus, Biratnagar for providing lab facilities. We are grateful to AquaFish Innovation Lab for financial support. 


\section{References}

APHA 1976. Standard methods for examination of water and waste water. American Public Health Association Inc., New York.

APHA 1985. Standard methods for examination of water and waste water $\left(16^{\text {th }}\right.$ ed.). American Public Health Association, American Water Works Association 1015, Fifteenth street NW, Washington D.C. $1268 \mathrm{p}$.

APHA 2005. Standard methods for examination of water and waste water. American Public Health Association, Washington: Byrd Prepress Springfield.

APHA 2012. Standard methods for examination of water and waste water ( $22^{\text {nd }}$ ed.), American Public Health Association, Washington.

Boyd, C.E. and C.S. Tucker 2014. Hand book for water quality. Alabama Agriculture Experiment Station, Auburn University, Alabama. pp. 237251.

Costa, P.R. 2014. Impact and effects of paralytic shellfish poisoning toxins derived from harmful algal blooms to marine fish. John Wiley and Sons Ltd.

Costa, P.R. and S. Garrido 2004. Domoic acid accumulation in the sardine Sardina pilchardus and its relationship to Pseudonitzschia diatom ingestion. Marine Ecology Progress Series 284: 261-268.

Guiry, M.D. and G.M. Guiry 2016. Algae Base. World-wide electronic publication, National. University of Ireland, Galway. http://www.algae base.org

Hosmani, S.P. 1988. Seasonal changes in phytoplankton communities in a freshwater pond at Dharwar, Karnatak State,India. Phykos 27: 82-87.

Lopez, C.B., E.B. Jewett, Q. Dortch, B.T. Walton and H.K. Hudnell 2008. Scientific assessment of freshwater harmful algal blooms. Interagency Working Group on Harmful Algal Blooms, Hypoxia, and Human Health of the Joint Subcommittee on Ocean Science and Technology. Washington, DC. 65p.

Mishra, S.R. and D.N. Saksena 1993. Photo planktonic composition of sewage polluted Morar
(Kalpi) River in Gwalior, Madhya Pradesh. Environ. Ecol. 11: 625-629.

Munawar, M. 1970. Limnological studies on the fresh water ponds of Hyderabad, India. The Biocenose-Distribution of unicellular and colonial phytoplankton in polluted and unpolluted environments. Hydrobiologia 35: 127-162.

Nwankwo, D.I. 1995. Euglenoids of some polluted storm-water channels in Lagos, Nigeria. Trop. Freshwater Biol. 4: 29-39.

Ohio EPA 2013. Seeing red: Emerging harmful algal blooms. Newsletter for Ohio's Public Drinking Water Systems. Spigot News $\sim$ summer 2013 Page 2.

Prescott, G.W. 1951. Algae of the western great lakes area. WM.C. Brown Publishers, Dubuque, Iowa. 977p.

Rahman, M.M., M.A. Jewell, S.S. Khan and M.M. Haque 2007. Study of euglenophytes bloom and its impact on fish growth in Bangladesh. Algae 22(3): 185-192

Rai, S.K. and R.K. Rai 2007. Some euglenophycean algae from Biratnagar, Nepal. Our Nature 5: 6066

Rehman, S.U. 1998. A red bloom of Euglena shafiqii, a new species, in Dal Lake, Srinagar, Kashmir. Water Air and Soil Pollution 108: 69-82.

Wild, A., J. Lee-Kaden, H. Gimmler and W. Simonis 1995. Phycological and hydro-chemical investigations of two polytrophic ponds near to an urban development. Limnology 25: 229-236.

Xavier, M.B., C.S.R. Mainardes, Pinto and M. Takino 1991. Euglena sanguinea Ehrenberg bloom in a fish-breeding tank (Pindamonhangaba, Sao Paulo, Brazil). Archive-fur- Hydrobiology. Supplement band, Algological Studied 62: 133-142

Zimba, P.V., M. Rowan and R.E. Triemer 2004. Identification of euglenoid algae that produce ichthyotoxin(s). Journal of Fish Diseases 27: 115117.

Zimba, P.V., P.D. Moeller, K. Beauchesne, H.E. Lane and R.E. Triemer 2010. Identification of euglenophycin- A toxin found in certain euglenoids. Toxicon 55(1): 100-104. Doi 10.1016/j.toxico n.2009.07.004 